\title{
Gedanken an ein Lebensende in Haft - eine Besonderheit der Inhaftierten im höheren Alter
}

\author{
Norman Meuschke' $\cdot$ Reinhold Jagsch ${ }^{2}$ \\ Eingegangen: 27. Juni 2020 / Angenommen: 10. Juli 2020 / Online publiziert: 23. Juli 2020 \\ (c) Der/die Autor(en) 2020
}

\section{Zusammenfassung}

Ziel der Studie war es, die Rolle etwaiger Gedanken an den Tod und das Sterben im Hinblick auf die Persönlichkeit, die physische und psychische Gesundheit sowie die Zufriedenheit mit unterschiedlichen Bereichen des Lebens und der Haftsituation von Inhaftierten im Seniorenalter zu untersuchen. Die Studie wurde als fragenbogenbasierte Querschnittuntersuchung konzipiert. Insgesamt wurden 55 Inhaftierte im Alter von 60 Jahren und mehr aus 11 österreichischen Justizanstalten mittels Beck-Depression-Inventar II, Brief Symptom Inventar-53 und NEO-Fünf-Faktoren-Inventar untersucht. Zusätzlich wurde die Zufriedenheit mit unterschiedlichen Facetten ihres aktuellen Lebens und Alltages mittels visueller Analogskalen (VAS) bewertet. Die statistische Überprüfung etwaiger Unterschiede erfolgte anhand von Mittelwertsvergleichen.

Die Ergebnisse weisen darauf hin, dass inhaftierte Senioren, die keine Gedanken an den Tod berichten, niedrigere Neurotizismuswerte und eine deutlich geringere psychische Belastung berichten als Senioren, die derartige Gedanken angaben. Zudem wird deutlich, dass sich die Subgruppen anhand der Dauer der derzeitigen Inhaftierung unterscheiden, wobei die Intensität der Gedanken an den Tod mit der Haftdauer signifikant zunimmt. Zudem ergab sich hinsichtlich des subjektiven physischen Gesundheitszustandes ein signifikanter Unterschied zwischen den beiden Subgruppen, wohingegen die derzeitige Haftsituation sowie der objektive Gesundheitszustand keinen Einfluss zu haben scheinen.

Schlüsselwörter Ältere Inhaftierte · Gedanken an den Tod · Persönlichkeit · Psychische Belastung · Gesundheitszustand

\section{Thoughts of dying in prison-a peculiarity of elderly inmates}

\begin{abstract}
The aim of the study was to examine the role of thoughts of death and dying with respect to the personality, the physical and psychological conditions and the satisfaction with different aspects of life and prison environment of aged inmates. The study was designed as a cross-sectional questionnaire study using the German versions of the Beck depression inventory II, the brief symptom inventory 53 and the NEO five-factor inventory. Additionally, visual analogue scales (VAS) were used to collect data on satisfaction with different aspects of life and the prison environment. In total 55 inmates aged 60 years and older from 11 Austrian correctional facilities were included. Comparisons of means were primarily used to examine possible differences between the subgroups. The results clearly indicate that older inmates who do not suffer from thoughts of death and dying show significantly lower neuroticism scores and less psychological distress, when compared to inmates reporting these thoughts. Furthermore, thoughts of death and dying are intensified in the course of the increasing duration of the present imprisonment. Additionally, older inmates with thoughts of death and dying reported a significantly lower satisfaction with their subjective physical condition, whereas the conditions within the correctional facilities as well as the objective health condition seem to have no influence.
\end{abstract}

Keywords Older inmates $\cdot$ Thoughts of death and dying $\cdot$ Personality $\cdot$ Psychological distress $\cdot$ State of health

Mag. Norman Meuschke normanmeuschke@gmx.net

Mag. Dr. Reinhold Jagsch reinhold.jagsch@univie.ac.at
1 Justizanstalt Asten, Technologiestraße 5, 4481 Asten, Österreich

2 Institut für klinische und Gesundheitspsychologie, Fakultät für Psychologie, Universität Wien, Liebiggasse 5, 1010 Wien, Österreich 


\section{Theoretischer Hintergrund}

Bereits seit mehreren Jahren ist bekannt, dass sich der demographische Wandel hin zu einer immer älter werdenden Gesellschaft auch in den internationalen Gefangenenpopulationen zunehmend widerspiegelt, sodass die Inhaftierten im Alter von 50 Jahren und mehr derzeit weltweit die am schnellsten wachsende Insassengruppe darstellt (Baidawi et al. 2011; ACLU 2012; Hayes et al. 2013; Marti et al. 2014; Baidawi und Trotter 2015). Betrachtete man noch vor wenigen Jahren die deutschsprachige medizinische und psychologische Literatur, die sich mit älteren Menschen im Strafvollzug befasste, so zeigte sich, dass dieses Thema im vergangenen Forschungsgeschehen kaum Beachtung gefunden hat.

In den letzten zwei Jahren konnte jedoch eine deutliche Zunahme des Interesses an Inhaftierten im höheren Alter festgestellt werden. Dies spiegelt sich nicht nur in einer vermehrten medialen Präsenz dieser Thematik (u.a. Bock 2018) und in einer Zunahme der seniorenspezifischen Unterbringungsformen und Angebote innerhalb von Justizanstalten (Kenkmann et al. 2020) wider, sondern auch in einer steigenden Anzahl an Publikationen zu diesem Thema im deutschsprachigen Raum. Jedoch handelt es sich dabei zumeist um qualitative Befragungen dieser Population und Berichte bezüglich der praktischen Umsetzung von seniorenspezifischen Abteilungen, Haftanstalten und Interventionen (u.a. Friedrichs 2012; Allgöwer 2013; Hirschbeck 2013; Graber et al. 2014; Ast 2015; Berger-Zell 2019; Ghanem und Kenkmann 2019), wobei zunehmend auch quantitative Erhebungen publiziert werden (u. a. Meyer 2019).

Ein Aspekt, der insbesondere im Rahmen der qualitativen Untersuchungen von inhaftierten Senioren häufig thematisiert wird, sind die Gedanken an ein mögliches Lebensende in Haft (u. a. Handtke und Wangmo 2014; Marti 2015; Shaw und Elger 2016; Lob-Hüdepohl 2019; Hostettler et al. 2019). Neben rechtlichen und palliativen Aspekten sowie der Frage nach einem würdevollen Abschied vom sozialen Umfeld (Hostettler et al. 2017; Richter et al. 2017; Brägger 2018) wird aufgrund der in den letzten Jahren stetig liberalisierten Gesetzgebung im Bereich der aktiven Sterbehilfe insbesondere die Übertragbarkeit dieses Rechts auf den Haftkontext international kontrovers diskutiert (Handtke und Wangmo 2014; Marti 2015; Willems 2015; Devolder 2016; Shaw und Elger 2016; Walcher 2018).

Grundsätzlich weisen die bisherigen Forschungsergebnisse zum Thema Angst vor dem Tod darauf hin, dass diese in eine bewusste und eine unbewusste Komponente unterteilt werden kann, wobei beide Anteile negativ miteinander korreliert sind und auch mit dem Lebensalter in enger Verbindung stehen (Hayslip 2003). So fanden Galt und Hayslip (1998) eindeutige altersspezifische Unterschiede, die darauf hinweisen, dass bei jungen Probanden die To- desangst primär im Unbewussten liegt, wohingegen über 60-jährige Probanden eine höhere bewusste Todesangst berichten. Die Autoren postulieren zudem, dass die kumulierten Verlusterlebnisse im Laufe des Lebens dazu beitragen, dass eine bewusstere Auseinandersetzung mit dem eigenen Tod stattfinden kann (Hayslip 2003). Bereits 2018 wurde in einer eigenen Publikation, deren primärer Fokus auf den soziodemographischen und delinquenzspezifischen Charakteristika der Inhaftierten im Alter von 60 Jahren und mehr sowie ihrer Haftsituation lag, darauf hingewiesen, dass den Gedanken an das Lebensende innerhalb dieser Altersgruppe eine besondere Bedeutung zukommt.

Hinsichtlich etwaiger Einflussfaktoren auf die Angst vor dem Tod kam Aday (2006b) im Zuge einer Untersuchung in einem amerikanischen Hochsicherheitsgefängnis zu dem Schluss, dass innerhalb der Gruppe der Inhaftierten ab 50 Jahren insbesondere eine abnehmende physische und psychische Gesundheit sowie soziale Unterstützung als auch ein höheres Alter sowie vermehrte Sorgen um die eigene Sicherheit mit einer steigenden Todesangst in Verbindung stehen. Aus Untersuchungen in der Allgemeinbevölkerung ist darüber hinaus bekannt, dass Zusammenhänge zwischen der Todesangst und gewissen Persönlichkeitsdimensionen bestehen. Vor allem hinsichtlich des Neurotizismus finden sich verschiedenste internationale Studien, die auf eine positive Korrelation dieser Persönlichkeitsdimension mit der Angst vor dem Tod hinweisen (Frazier und Foss-Goodmann 1989; Cully et al. 2001; Jastrzebski und Slaski 2011). Ebenso finden sich Hinweise auf negative Zusammenhänge zwischen Todesangst und Offenheit für neue Erfahrungen (Cully et al. 2001; Jastrzebski und Slaski 2011), Verträglichkeit (Cully et al. 2001), Gewissenhaftigkeit (Jastrzebski und Slaski 2011) sowie der Extraversion (Frazier und Foss-Goodmann 1989; Cully et al. 2001; Szaniawski 1998), wobei eine Studie an 18- bis 23-jährigen Studenten positive Korrelationen bezüglich dieser Persönlichkeitsdimension ergab (Jastrzebski und Slaski 2011). Im Rahmen der Untersuchung der Zusammenhänge zwischen Spiritualität, Lebenszufriedenheit und Angst vor dem Tod bei über 60-jährigen Probanden konnte zudem gezeigt werden, dass Lebenszufriedenheit und Angst vor dem Tod nicht nur negativ korreliert sind (Given und Range 1990; Roshani 2012; Taghiabadi et al. 2017), sondern dass die Lebenszufriedenheit auch einen besseren Prädiktor der Todesangst darstellt als die Religiosität (Roshani 2012).

Im deutschsprachigen Raum wurden die Gedanken an den Tod von Inhaftierten bisher noch in keiner empirischen Studie thematisiert, zumal sich die Untersuchungen innerhalb von Gefangenenpopulationen zumeist auf Suizidalität beziehen (u. a. Pesch 2015), die jedoch von bloßen Gedanken an ein natürliches Lebensende aufgrund des zunehmenden Alters zu unterscheiden ist. 
Auf Basis dieser bisherigen Erkenntnisse war somit das Ziel der Studie, den Zusammenhang zwischen den Gedanken an das Lebensende, der psychischen und physischen Gesundheit sowie der Haftsituation von Inhaftierten im Seniorenalter erstmalig im deutschsprachigen Raum einer empirischen Untersuchung zu unterziehen.

\section{Stichprobe}

Die Daten wurden in 11 zentral- und ostösterreichischen Justizanstalten erhoben, wobei sowohl Anstalten zum Vollzug von Untersuchungshaften und kurzen Freiheitsstrafen als auch Anstalten für den Vollzug von mittellangen und zeitlich unbegrenzten Freiheitsstrafen inkludiert wurden. Die Studie wurde von den Ethikkommissionen des Österreichischen Ministeriums für Justiz sowie der Bundesländer Wien, Niederösterreich und Oberösterreich genehmigt. Inhaftierte, die sich im Maßnahmenvollzug (gem. § 21 öStGB) oder der vorläufigen Anhaltung dafür ( $§ 429$ und 430 öStPO) befanden oder psychische Auffälligkeiten gemäß § 129 öStVG aufwiesen, wurden aufgrund ihrer besonderen Betreuungsbedürfnisse und der teilweise separaten Unterbringung von der Untersuchung ausgeschlossen.

\section{Datenerhebung}

Die Datenerhebung erfolgte einerseits unter Verwendung dreier psychologischer Testinstrumente, um die psychischen Belastungsmomente sowie die Persönlichkeitsmerkmale der Studienteilnehmer zu erfassen. Darüber hinaus wurden die Zufriedenheit mit unterschiedlichen Bereichen des Lebens und der Haftsituation sowie biografische Informationen anhand selbstentwickelter Fragebögen erhoben. Die gesamte Bearbeitungsdauer betrug zwischen 60 und 90 min. Aufgrund dieses Designs konnten Personen mit nicht ausreichenden Sprachkenntnissen nicht an der Studie teilnehmen und sind daher innerhalb der Stichprobe unterrepräsentiert.

\section{Instrumente und Kennwerte}

Die Persönlichkeitscharakteristika der Probanden wurden anhand der deutschen Version des NEO-Fünf-FaktorenInventars (NEO-FFI, Borkenau und Ostendorf 1993) ermittelt. Die Ausprägung der fünf Persönlichkeitsdimensionen Neurotizismus, Extraversion, Offenheit für Erfahrungen, Verträglichkeit sowie Gewissenhaftigkeit wird dabei anhand von 60 Items mittels einer fünfstufigen Skala erhoben. Im Bereich der psychischen Belastungsmomente wurde das Ausmaß der depressiven Symptome mittels der deutschen
Version des Beck-Depressions-Inventars II (BDI-II, Hautzinger et al. 2009) erfasst. Basierend auf dem DSM-IV wird darin das Vorliegen von 21 depressiven Symptomen anhand einer vierstufigen Skala bewertet. Summenwerte niedriger als 14 werden als unauffällig, Werte zwischen 14 und 19 als leichtes, Werte zwischen 20 und 28 als mittleres und Werte höher als 28 als schweres depressives Syndrom gewertet. Ergänzend wurde ein breiteres Spektrum an psychischen Beeinträchtigungen unter Verwendung der deutschen Version des Brief Symptom Inventory in der 53-Item-Version (BSI-53, Franke 2000) erhoben. Dabei werden die Symptome von neun möglichen psychischen Störungsbildern (BSI1: Somatisierung; BSI2: Zwanghaftigkeit; BSI3: Unsicherheit im Sozialkontakt; BSI4: Depressivität; BSI5: Ängstlichkeit; BSI6: Aggressivität/Feindseligkeit; BSI7: Phobische Angst; BSI8: Paranoides Denken; BSI9: Psychotizismus) anhand von 49 der 53 Items auf einer fünfstufigen Skala bewertet. Unter Hinzunahme der vier übrigen Items, die sich auf einzelne Beschwerden (schlechter Appetit, Einschlafschwierigkeiten, Gedanken an den Tod und ans Sterben, Schuldgefühle) beziehen, lässt sich ein Gesamtwert berechnen, der Global Severity Index (GSI). Bei der Auswertung werden die Rohwerte in T-Werte umgewandelt, wobei ein T-Wert von 63 oder mehr entweder hinsichtlich des Gesamtwertes oder von zwei Subskalen auf klinische Relevanz hinweist. Darüber hinaus wurde der Mini-Mental-Status-Test (MMST), entnommen aus der CERAD-Testbatterie (Thalmann et al. 1997), durchgeführt. Dabei handelt es sich um das gängigste Verfahren zur Durchführung eines Demenzscreenings. Wird dabei der Cut-off-Wert von 24 (von 30) Punkten unterschritten, kann mit hoher Sicherheit von einem kognitiven Abbau im Sinne einer Demenz ausgegangen werden (Creavin et al. 2016). Die Variablen hinsichtlich der anhand von visuellen Analogskalen (VAS) bewerteten Zufriedenheit mit unterschiedlichen Aspekten des Lebens und der Haft sowie des Gesundheitszustands der älteren Inhaftierten wurden aus einer weitergefassten Erhebung, die die altersspezifischen soziodemografischen und kriminologischen Charakteristika der österreichischen Insassenpopulation im Allgemeinen sowie die Haftsituation der älteren Inhaftieren im Speziellen im Fokus hat (Meuschke 2018), entnommen.

\section{Datenauswertung}

Die statistische Analyse erfolgte unter Verwendung von IBM SPSS für Windows (Version 25). Für deskriptive Statistiken wurden absolute und relative Häufigkeiten angegeben, wobei die Summenwerte, aufgrund von einzelnen fehlenden Daten, nicht immer dem Umfang der Gesamtstichprobe entsprechen müssen. Zur Beschreibung von stetigen Variablen werden Mittelwert (M), Standardabweichung 
(SD) und Spannweite (Min; Max) angegeben. Im Falle von schief verteilten Variablen wurde darüber hinaus der Median (Mdn) angegeben. Während die Vergleiche von kategorialen Variablen mittels $C h i^{2}$-Test durchgeführt wurden, kam bei der Untersuchung der altersspezifischen Unterschiede die einfaktorielle Varianzanalyse (ANOVA) zum Einsatz. Darüber hinaus wurden für signifikante Ergebnisse ergänzend Effektstärken angeführt (partielles $E t a^{2}$ ). Im Einklang mit den Standards wurde ein $E t a^{2}>0,01$ als klein, ein $E t a^{2}>0,06$ als moderat und ein $E t a^{2}>0,14$ als ein großer Effekt bewertet (Cohen 1988). Des Weiteren wurden Posthoc-Tests durchgeführt, um signifikante Mittelwertsunterschiede zwischen den untersuchten Subgruppen zu identifizieren. Bei den Vergleichen zwischen älteren Inhaftierten mit bzw. ohne Gedanken an den Tod kam der t-Test für unabhängige Stichproben zur Anwendung. Im Falle signifikanter Ergebnisse wurde die Effektstärke mittels Cohen's $d$ angegeben. Anhand der Standards wird dabei ein Wert von $>0,20$ als kleiner, ein Wert von >0,50 als moderater und ein Wert von >0,80 als großer Effekt angesehen (Cohen 1988). Das Signifikanzniveau wurde auf $\alpha \leq 0,05$ festgelegt, wobei hinsichtlich des Intragruppenvergleiches der älteren Inhaftierten auch tendenziell signifikante Ergebnisse $(\alpha \leq 0,10)$ interpretiert werden.

\section{Stichprobenbeschreibung}

Insgesamt haben 55 Inhaftierte aus 11 Justizanstalten die zur Verfügung gestellten Fragebögen bearbeitet. Das Durchschnittsalter betrug 66,49 Jahre $\quad(\mathrm{SD}=5,32$;
Range $=60 ; 82)$. Der überwiegende Anteil dieser Gruppe war in seinen 60ern $(n=44 ; 80,0 \%), 10$ Inhaftierte waren in ihren 70ern $(18,2 \%)$ und ein Proband sogar über 80 Jahre alt $(1,8 \%)$. Das Durchschnittsalter bei der ersten strafrechtlichen Verurteilung lag bei 40,57 ( $\mathrm{SD}=20,13)$ Jahren, mit einer großen Spannweite von 10 bis 73 Jahren. Die Anzahl der Vorverurteilungen belief sich im Mittel auf 8,00 $(\mathrm{SD}=9,24 ; \mathrm{Mdn}=4$; Range $=0 ; 36)$ und war damit ebenso weit gestreut wie die Anzahl der bisherigen Inhaftierungen, für die sich ein Durchschnittswert von 5,33 ( $\mathrm{SD}=6,28$; Mdn $=2$; Range $=1 ; 33$ ) ergab. Zum Erhebungszeitpunkt waren die Probanden durchschnittlich bereits 65,71 Monate $(\mathrm{SD}=108,88 ; \mathrm{Mdn}=23)$ inhaftiert, wobei die kürzeste Zeitspanne zwei Monate und die längste Zeitspanne mehr als 40 Jahre betrug. Weiterführende Charakteristika der Stichprobe können Tab. 1 entnommen werden.

Grundsätzlich zeigte sich hinsichtlich eines Vergleiches der Seniorenstichprobe mit einer unter 40-jährigen $(M=29,33 ; \mathrm{SD}=5,70)$ und einer 40- bis 59-jährigen Vergleichsgruppe $(M=48,41 ; \mathrm{SD}=5,51)$, der im Rahmen einer eigenen Voruntersuchung (Meuschke 2018) durchgeführt wurde, dass die Inhaftierten im Alter von 60 Jahren und mehr tendenziell signifikant mehr Gedanken an den Tod und das Sterben (BSI Item 39) berichten $(F[2,173]=2,144$, $\left.p=0,053, E t a^{2}=0,033\right)$ als die unter 40-jährigen Inhaftierten $(p=0,050)$. Basierend auf dem Vorliegen von Gedanken an den Tod und das Sterben (BSI Item 39) wurde die Stichprobe der Senioren in eine Subgruppe, die keine Gedanken an den Tod und das Sterben berichtete $(n=30 ; 54,6 \%)$, und eine Teilgruppe, die derartige Gedanken in der Erhebung angab $(n=25 ; 45,4 \%)$, unterteilt.

Tab. 1 Beschreibung der Stichprobe $(N=55)$

\begin{tabular}{|c|c|c|c|c|c|c|c|c|c|c|c|c|c|}
\hline & $n$ & $\%$ & ${ }^{\mathrm{a}} n_{\mathrm{kG}}$ & $\mathrm{a} \%$ & ${ }^{\mathrm{b}} n_{\mathrm{G}}$ & b\% & & $n$ & $\%$ & ${ }^{\mathrm{a}} n_{\mathrm{kG}}$ & a\% & $\mathrm{b}_{n_{\mathrm{G}}}$ & $\mathrm{b} \%$ \\
\hline \multicolumn{7}{|l|}{ Beziehungsstatus } & \multicolumn{7}{|l|}{ Kinder } \\
\hline Ledig & 6 & 11 & 2 & 6,5 & 4 & 16 & $\mathrm{Ja}$ & 12 & 22 & 5 & 17 & 7 & 28 \\
\hline In Partnerschaft & 8 & 15 & 3 & 10 & 5 & 20 & Nein & 43 & 78 & 25 & 83 & 18 & 72 \\
\hline Verheiratet & 9 & 16 & 5 & 17 & 4 & 16 & - & - & - & - & - & - & - \\
\hline Geschieden & 28 & 51 & 18 & 60 & 10 & 40 & - & - & - & - & - & - & - \\
\hline Verwitwet & 4 & 7 & 2 & 6,5 & 2 & 8 & - & - & - & - & - & - & - \\
\hline \multicolumn{7}{|c|}{ Höchster Schulabschluss } & \multicolumn{7}{|c|}{ Beruf erlernt } \\
\hline Keiner & 3 & 5 & 1 & 3 & 2 & 8 & $\mathrm{Ja}$ & 39 & 71 & 23 & 77 & 16 & 64 \\
\hline Hauptschule & 13 & 24 & 5 & 17 & 8 & 32 & Nein & 15 & 27 & 6 & 20 & 9 & 36 \\
\hline Berufsschule & 27 & 49 & 15 & 50 & 12 & 48 & Fehlend & 1 & 2 & 1 & 3 & 0 & - \\
\hline Matura & 7 & 13 & 5 & 17 & 2 & 8 & - & - & - & - & - & - & - \\
\hline Universität & 1 & 2 & 1 & 3 & 0 & 0 & - & - & - & - & - & - & - \\
\hline Fehlend & 4 & 7 & 3 & 10 & 1 & 4 & - & - & - & - & - & - & - \\
\hline \multicolumn{7}{|c|}{ Art des Anlassdeliktes } & \multicolumn{7}{|c|}{ Geburtsland } \\
\hline $\begin{array}{l}\text { Gewalt-/ } \\
\text { Sexualstraftat }\end{array}$ & 29 & 53 & 13 & 43 & 16 & 64 & Österreich & 39 & 71 & 20 & 67 & 19 & 76 \\
\hline Andere & 26 & 47 & 17 & 57 & 9 & 36 & Anderes & 16 & 29 & 10 & 33 & 6 & 24 \\
\hline
\end{tabular}

${ }^{\mathrm{a}} n_{\mathrm{kG}}$ : Senioren ohne Gedanken an den Tod

$\mathrm{b}_{n_{\mathrm{G}}}$ : Senioren mit Gedanken an den Tod 


\section{Ergebnisse des Vergleiches der inhaftierten Senioren mit und ohne Gedanken an den Tod}

Nachdem die Gruppe der Inhaftierten im Seniorenalter anhand der selbstberichteten Gedanken an den Tod in zwei Gruppen geteilt wurde, wurden die Charakteristika der Delinquenz sowie die Ergebnisse der erhobenen Verfahren miteinander verglichen. Die Betrachtung der Aspekte der Delinquenz weist auf wesentliche Unterschiede zwischen den zwei Gruppen hinsichtlich der Dauer der gegenwärtigen Inhaftierung hin, wobei länger inhaftierte Senioren häufiger Gedanken an den Tod und das Sterben berichten. Damit einhergehend zeigt sich auch, dass diese Senioren bei der Begehung ihres Anlassdeliktes zumeist jünger waren als die Inhaftierten, die sich nicht mit Gedanken an den Tod beschäftigen (Tab. 2).

Hinsichtlich eines etwaigen kognitiven Abbaus, erhoben mittels MMST, ergab sich kein Unterschied zwischen Senioren ohne $(M=26,77 ; \mathrm{SD}=2,46)$ und mit Gedanken an den Tod $(M=27,17 ; \mathrm{SD}=1,83)$, wobei sich in beiden Gruppen keine kognitiven Beeinträchtigungen im Sinne einer be- ginnenden oder fortgeschrittenen Demenz fanden. Jedoch ergab sich in Bezug auf den BDI-II-Summenwert ein hochsignifikantes Resultat $(t[53]=-3,473 ; p=0,001 ; d=0,94)$, wobei die Subgruppe, die keine Gedanken an den Tod berichtete, einen laut Manual im unauffälligen Bereich liegenden Mittelwert von 10,93 ( $\mathrm{SD}=7,93)$ aufwies. In der Subgruppe mit Gedanken an den Tod fand sich hingegen ein Mittelwert von 19,08 (SD = 9,48), der einem Schweregrad an der Schwelle zu einem mittleren depressiven Syndrom entspricht. Des Weiteren ergab sich auch für den BSIGesamtwert ein signifikanter höherer Mittelwert für die älteren Inhaftierten mit Gedanken an den $\operatorname{Tod}(t[53]=-4,493$; $p<0,001 ; d=1,22)$. Neben allen Subskalen des Verfahrens, ausgenommen die Skala Phobische Angst (BSI7), zeigten auch die Zusatzitems Einschlafschwierigkeiten und Schuldgefühle signifikante Unterschiede in gleicher Richtung. Dabei überschritt nicht nur der GSI den Grenzwert, der auf Behandlungsbedürftigkeit hinweist, sondern auch die Skalen Somatisierung (BSI1), Depressivität (BSI4), Paranoides Denken (BSI8) und Psychotizismus (BSI9) (Abb. 1).

Tab. 2 Vergleich hinsichtlich Alter und Aspekten der Delinquenz zwischen Inhaftierten ohne und mit Gedanken an den Tod

\begin{tabular}{|c|c|c|c|c|c|c|c|}
\hline & \multicolumn{2}{|c|}{ Keine Gedanken an Tod $(n=30)$} & \multicolumn{2}{|c|}{ Gedanken an Tod $(n=25)$} & \multirow[b]{2}{*}{$t$} & \multirow[b]{2}{*}{$p$} & \multirow[b]{2}{*}{$d$} \\
\hline & MW & SD & MW & SD & & & \\
\hline Alter & 66,47 & 5,27 & 66,52 & 5,48 & $-0,037$ & 0,971 & - \\
\hline Alter Erstdelikt & 41,32 & 19,97 & 39,72 & 20,67 & 0,287 & 0,776 & - \\
\hline Alter aktuelles Delikt & 62,71 & 8,40 & 57,08 & 13,03 & 1,891 & $0,064^{* *}$ & 0,520 \\
\hline Anzahl Vorstrafen & 7,72 & 8,37 & 8,88 & 10,31 & $-0,641$ & 0,524 & - \\
\hline Gefängnisaufenthalte & 5,50 & 6,83 & 5,13 & 5,70 & 0,213 & 0,832 & - \\
\hline Zeit in Haft (Monate) & 31,00 & 31,32 & 104,96 & 147,36 & $-2,360$ & $0,027^{*}$ & 0,715 \\
\hline
\end{tabular}

${ }^{*} p<0,05,{ }^{* *} p<0,10$

$M W$ Mittelwert, $S D$ Standardabweichung

Abb. 1 Mittelwerte (T-Werte) der Skalen des BSI von inhaftierten Senioren mit und ohne Gedanken an den Tod

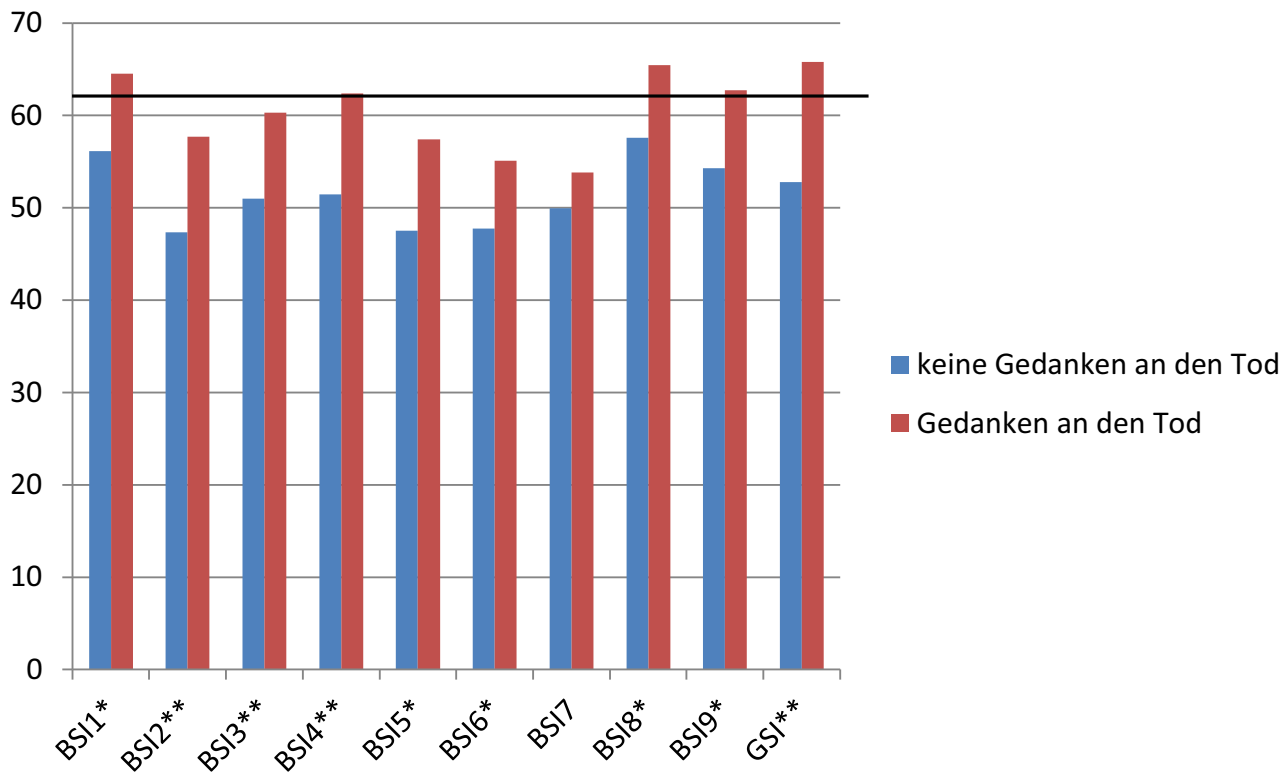


Tab. 3 Unterschiede hinsichtlich des subjektiven Gesundheitszustandes der älteren Inhaftierten

\begin{tabular}{|c|c|c|c|c|c|c|}
\hline & \multicolumn{2}{|c|}{ Keine Gedanken an Tod $(n=30)$} & \multicolumn{2}{|c|}{ Gedanken an Tod $(n=25)$} & \multirow[b]{2}{*}{$t$} & \multirow[b]{2}{*}{$p$} \\
\hline & $M W$ & $S D$ & $M W$ & $S D$ & & \\
\hline Allg. Gesundheitszustand & 48,70 & 32,59 & 37,52 & 26,51 & 1,377 & 0,174 \\
\hline \multicolumn{7}{|l|}{ Physische Beschwerden (0-5) } \\
\hline Gehbeschwerden & 1,50 & 1,82 & 2,17 & 1,90 & $-1,313$ & 0,195 \\
\hline Hörbeschwerden & 1,70 & 1,90 & 1,46 & 1,59 & 0,500 & 0,619 \\
\hline Sehbeschwerden & 2,13 & 1,74 & 2,04 & 1,46 & 0,207 & 0,837 \\
\hline \multirow[t]{2}{*}{ Schmerzen } & 2,47 & 1,83 & 2,58 & 1,79 & $-0,235$ & 0,815 \\
\hline & $n$ & $\%$ & $n$ & $\%$ & $C h i^{2}$ & $p$ \\
\hline Chronische Erkrankung & 15 & 50 & 17 & 68 & 1,816 & 0,178 \\
\hline Diät notwendig & 6 & 20 & 3 & 12 & 0,540 & 0,462 \\
\hline Verwendung von Hilfsmitteln & 26 & 87 & 21 & 84 & 0,657 & 0,720 \\
\hline
\end{tabular}

Im Bereich der Persönlichkeitsdimensionen fand sich für die Skala Neurotizismus ein signifikanter Unterschied zwischen Senioren ohne und mit Gedanken an den Tod, wobei Letztere höhere Werte erreichen $(t[53]=-2,441 ; p=0,018$; $d=0,66)$. Zudem berichteten diese Inhaftierten eine zumindest tendenziell signifikante geringere Extraversion $(t[53]=1,788 ; p=0,080 ; d=-0,49)$ sowie eine tendenziell geringere Gewissenhaftigkeit $(t[53]=1,867 ; p=0,067$; $d=-0,51)$. Obwohl sich eine zumindest tendenziell signifikante negative Korrelation zwischen den berichteten Gedanken an den Tod und der Einschätzung des eigenen Gesundheitszustandes innerhalb der Seniorengruppe findet
( $r=-0,226, p=0,097)$, macht die Betrachtung der in Tab. 3 dargestellten Ergebnisse deutlich, dass sich hinsichtlich des physischen Zustands keine wesentlichen Unterschiede zwischen Senioren mit und ohne Gedanken an den Tod ergeben.

Die abschließende Betrachtung der in Tab. 4 dargestellten Ergebnisse etwaiger Unterschiede hinsichtlich der erhobenen Zufriedenheitswerte macht deutlich, dass die Gedanken an den Tod und das Sterben nicht mit Aspekten der Haft in Verbindung stehen, sondern sich primär Unterschiede hinsichtlich der Zufriedenheit mit der finanziellen Situation außerhalb der Haftanstalt sowie mit dem eigenen

Tab. 4 Vergleich der Zufriedenheitswerte zwischen inhaftierten Senioren mit und ohne Gedanken an den Tod (VAS)

\begin{tabular}{|c|c|c|c|c|c|c|c|}
\hline \multirow[b]{2}{*}{ Zufriedenheit mit ... } & \multicolumn{2}{|c|}{ Keine Gedanken an Tod $(n=30)$} & \multicolumn{2}{|c|}{ Gedanken an Tod $(n=25)$} & \multirow[b]{2}{*}{$t$} & \multirow[b]{2}{*}{$p$} & \multirow[b]{2}{*}{$d$} \\
\hline & MW & SD & MW & SD & & & \\
\hline Leben im Allgemeinen & 61,13 & 28,71 & 52,76 & 30,63 & 1,045 & 0,301 & - \\
\hline Momentaner Situation in Haft & 34,67 & 29,76 & 31,72 & 31,00 & 0,359 & 0,721 & - \\
\hline $\begin{array}{l}\text { Momentaner Situation außer- } \\
\text { halb der Haftanstalt }\end{array}$ & 60,57 & 33,64 & 52,58 & 34,79 & 0,840 & 0,405 & - \\
\hline Alltag in Haft & 45,17 & 32,74 & 42,40 & 32,79 & 0,312 & 0,756 & - \\
\hline Finanzieller Situation in Haft & 44,73 & 37,73 & 52,92 & 38,25 & $-0,796$ & 0,429 & - \\
\hline $\begin{array}{l}\text { Finanzieller Situation außer- } \\
\text { halb der Haftanstalt }\end{array}$ & 58,11 & 31,42 & 34,33 & 29,75 & 2,787 & $0,004^{* *}$ & 0,78 \\
\hline $\begin{array}{l}\text { Momentaner familiäre Situa- } \\
\text { tion }\end{array}$ & 50,83 & 37,01 & 48,00 & 35,60 & 0,282 & 0,779 & - \\
\hline Beziehung zur Partnerin & 69,61 & 29,44 & 80,01 & 31,61 & $-0,947$ & 0,351 & - \\
\hline Beziehung zu Kindern & 58,56 & 40,92 & 61,40 & 36,86 & $-0,242$ & 0,810 & - \\
\hline Gefängnis insgesamt & 43,70 & 26,62 & 49,36 & 28,12 & $-0,771$ & 0,444 & - \\
\hline Ausgangsmöglichkeiten & 28,55 & 32,68 & 21,79 & 32,93 & 0,658 & 0,514 & - \\
\hline Besuchsmöglichkeiten & 50,89 & 35,58 & 44,65 & 37,52 & 0,608 & 0,546 & - \\
\hline Telefonmöglichkeiten & 65,83 & 29,03 & 59,09 & 38,21 & 0,724 & 0,473 & - \\
\hline Medizinischem Dienst & 50,52 & 35,52 & 52,28 & 36,85 & $-0,179$ & 0,859 & - \\
\hline Psychologischem Dienst & 80,96 & 20,75 & 61,29 & 42,30 & 2,061 & $0,047^{*}$ & 0,60 \\
\hline Sozialem Dienst & 69,07 & 24,66 & 60,43 & 36,87 & 0,969 & 0,339 & - \\
\hline Seelsorgerischem Dienst & 69,75 & 34,04 & 72,86 & 37,35 & $-0,281$ & 0,780 & - \\
\hline Gesundheitlicher Situation & 50,03 & 33,17 & 35,56 & 26,66 & 2,366 & $0,022^{*}$ & 0,64 \\
\hline
\end{tabular}

${ }^{* *} p<0,01,{ }^{*} p<0,05$ 
Gesundheitszustand ergaben. Die Mittelwerte der beiden Subgruppen weisen darauf hin, dass die Senioren, die Gedanken an den Tod berichteten, deutlich unzufriedener in diesen beiden Skalen sind als die Inhaftierten, die keine derartigen Gedanken berichteten. Es zeigten sich keinerlei Unterschiede hinsichtlich der Zufriedenheit mit der familiären Situation, insbesondere der Beziehung zu Kindern oder einer etwaigen Partnerin, die im Rahmen der Untersuchung ebenfalls erhoben wurden. Lediglich im Bereich der Betreuung der Fachdienste unterschieden sich die beiden Subgruppen signifikant voneinander, wobei die Probanden ohne Gedanken an den Tod zufriedener mit der Betreuung durch den Psychologischen Dienst waren als die Senioren, die Gedanken an den Tod berichteten.

\section{Diskussion}

In der vorliegenden Studie wurden 55 männliche Inhaftierte ab einem Alter von 60 Jahren aus 11 österreichischen Justizanstalten mittels Selbstbeschreibungsfragebögen untersucht. Insgesamt 30 Inhaftierte $(54,5 \%)$ berichteten, bislang keine Gedanken an den Tod und ans Sterben gehabt zu haben, während die restlichen 25 (45,5\%) sich bereits in unterschiedlicher Intensität damit beschäftigt haben. Beide Gruppen wiesen ein gleiches Alter auf, hatten die gleiche Anzahl von Vorstrafen und die gleiche Anzahl an bisherigen Gefängnisaufenthalten. Auch bei der Abfrage der Zufriedenheit mit diversen Aspekten, die die persönliche Situation in der Haft betreffen, unterschieden sich die beiden Gruppen lediglich marginal. Es konnten weder Unterschiede bezüglich der Zufriedenheit mit diversen Aspekten der Haft noch bezüglich der familiären Situation oder den Kontaktmöglichkeiten mit Angehörigen erhoben und somit der von Aday (2006b) beschriebene Einfluss der sozialen Unterstützung sowie der Haftbedingungen auf die Ausprägung der Todesangst in der vorliegenden Untersuchung nicht bestätigt werden. Dabei bieten der höhere Versorgungsstandard sowie die besseren Kontaktmöglichkeiten mit Angehörigen und Mitinsassen in österreichischen Haftanstalten als auch die altersadäquaten Gegebenheiten in Seniorenabteilung der Justizanstalt Suben (Oberösterreich) und der Lungenheilstätte Wilhelmshöhe (Außenstelle der Justizanstalt WienJosefstadt), in denen ein Teil der Stichprobe $(n=20 ; 36,3 \%)$ untergebracht war, mögliche Erklärungen für diesen Unterschied. Zudem wird in $\S 9$ Abs 3 öStVG geregelt, dass der Freiheitsentzug möglichst nah am Wohnort vollzogen werden sollte, was die Aufrechterhaltung sozialer Bindung erleichtert.

Hinsichtlich der Gedanken an den Tod und das Sterben unterstreichen die vorliegenden Ergebnisse die in qualitativen Untersuchungen (u.a. Handtke und Wangmo 2014; Marti 2015; Shaw und Elger 2016; Lob-Hüdepohl 2019;
Hostettler et al. 2019) beobachtete Bedeutung dieser Gedanken in der Gruppe der Inhaftierten im höheren Alter, wobei dieser Unterschied durch die Erhebung der bewussten Gedanken an den Tod, die vor allem im höheren Alter von besonderer Bedeutung sind (Galt und Hayslip 1998), begünstigt sein könnte. Dabei scheinen insbesondere zeitbezogene Aspekte eine Rolle zu spielen, was im Gegensatz zu den Ergebnissen von Pesch (2015) und Aday (2006b) steht, die in ihren Untersuchungen keinen Zusammenhang zwischen der Dauer der Inhaftierung und möglicher Suizidalität von Inhaftierten bis 60 Jahren bzw. Todesangst von Inhaftierten ab 50 Jahren fanden. In der vorliegenden Studie zeigen Senioren, deren aktuelle Inhaftierung durchschnittlich vor fast zehn Jahren begann und deren Haftdauer durchschnittlich fast noch weitere zehn Jahre andauern wird, eine deutlich höhere Neigung, sich mit Gedanken an einen Tod in der Anstalt zu beschäftigen, als Inhaftierte, die aktuell deutlich später inhaftiert wurden und deren Haftende absehbar ist. Der Anteil von Probanden mit schweren Gewaltoder Sexualdelikten in der betroffenen Teilpopulation mit Gedanken an den Tod sowie die lange Inhaftierungsdauer legen dabei den Schluss nahe, dass diese tatsächlich einen Tod in Haft erleiden könnten.

Dabei fanden sich in der vorliegenden Untersuchung, im Gegensatz zur bisherigen Literatur (Given und Range 1990; Roshani 2012; Taghiabadi et al. 2017), keine Hinweise auf eine grundsätzlich reduzierte Lebenszufriedenheit in der Gruppe der Senioren mit Gedanken an den Tod, jedoch weisen diese eine geringere Zufriedenheit mit ihrer finanziellen Situation außerhalb der Haft auf. Dies könnte einerseits auf die längere Haftdauer innerhalb dieser Subgruppe, die sowohl die Zeitspanne der möglichen Erwerbsfähigkeit reduziert als auch mit einem stetigen Wegfall der sozialen und somit auch der finanziellen Unterstuitzung (Aday 2006a) einhergeht, zurückzuführen sein. Andererseits stellt eine mögliche Beerdigung eine besondere finanzielle Belastung dar. Sollten die Kosten nicht von Angehörigen übernommen werden, so erfolgt zumeist eine Sozialbestattung, bei der den Verstorbenen jedoch kaum Mitspracherecht bezüglich der Bestattungsmodalitäten eingeräumt wird. Nicht nur stellt die Angst vor einer anonymen Bestattung in einem Gefängnisfriedhof bzw. einem Gemeinschaftsgrab an sich schon ein Belastungsmoment dar (Leigey 2015), sondern es kommt auch zu einer drastischen Einschränkung der Selbstbestimmung am Lebensende, die für viele betroffene Inhaftierte von besonderer Wichtigkeit ist (Marti 2015). Um diese Selbstbestimmung aufrechtzuerhalten, sind somit gewisse finanzielle Ressourcen außerhalb der Haftanstalt notwendig, sodass die Betroffenen sich im Prozess der Auseinandersetzung mit dem eigenen Lebensende in Haft auch mit ihrer defizitären finanziellen Lage konfrontiert sehen.

Betrachtet man die Ergebnisse bezüglich des körperlichen Gesundheitszustandes, so weisen diese nicht auf 
eine Unterschiedlichkeit der beiden Teilgruppen hin, weder wenn man den allgemeinen Gesundheitsstatus betrachtet, noch wenn einzelne Teilaspekte getrennt abgefragt werden. Somit finden sich zwar keine Hinweise auf einen Zusammenhang von objektivem Gesundheitszustand und Todesangst im höheren Alter, wie dies von Aday (2006b) beschrieben wurde. Jedoch wird der ebenfalls dort beschriebene Zusammenhang mit der subjektiven Einschätzung des Gesundheitszustandes in der vorliegenden Untersuchung bestätigt. Damit einhergehend ist es auf der Ebene von Persönlichkeitsdimensionen vornehmlich der Aspekt Neurotizismus, der eine Differenzierung der Senioren ermöglicht, wobei bereits Frazier und Foss-Goodmann (1989) postulierten, dass es sich bei dieser Dimension um den besten Prädiktor von Todesangst handele. Inhaftierte mit Gedanken an das Sterben berichten daher vermehrte Sorgen sowie negative Gedanken und Gefühlszustände und sind zudem leichter aus dem seelischen Gleichgewicht zu bringen (Borkenau und Ostendorf 1993). Darüber hinaus ergab sich eine tendenziell signifikant reduzierte Extraversion, was ebenso wie die negative Korrelation von Todesangst und Gewissenhaftigkeit bereits für nichtinhaftierte Populationen beschrieben wurde (Frazier und Foss-Goodmann 1989; Cully et al. 2001). Damit scheinen die vorliegenden Ergebnisse die Altersspezifität der von Jastrzebski und Slaski (2011) gefundenen positiven Korrelation zwischen Extraversion und Todesangst zu bestätigen. Hinsichtlich der Persönlichkeitsdimensionen Offenheit für Erfahrungen und Verträglichkeit konnte der in einigen Studien (Cully et al. 2001; Jastrzebski und Slaski 2011) beschriebene Zusammenhang mit ängstlichen Gedanken an den Tod nicht repliziert werden. Dies könnte auf die deutlich differenzierte Erhebung der Todesangst mittels eines speziellen Verfahrens (v. a. Death Anxiety Scale; Abdel-Khalek und Neimeyer 2017) in den Vergleichsuntersuchungen zurückzuführen sein, da sich zum Teil Zusammenhänge mit einzelnen Teilfacetten der Todesangst, nicht aber dem Gesamtwert ergaben.

Eindeutig hingegen sind die Unterschiede in den klinischen Testverfahren, die Beeinträchtigungen im Bereich der psychischen Gesundheit erheben. Dabei machen die Ergebnisse der vorliegenden Untersuchung im Einklang mit den bisherigen empirischen Ergebnissen zu diesem Thema (Aday 2006b) deutlich, dass ältere Probanden ohne Gedanken an den Tod signifikant weniger psychisch belastet sind als Inhaftierte, die derartige Gedanken berichten. Im Detail zeigen diese sowohl signifikant höhere Depressionswerte als auch höhere Beeinträchtigungen in den Subskalen des Brief Symptom Inventory, die in den Skalen Somatisierung, Depressivität, Paranoides Denken und Psychotizismus sogar Werte für Behandlungsbedürftigkeit erreichen. AbdelKhalek (2005) hebt zudem den Zusammenhang von generalisierter Angst und Angst vor dem Tod hervor, der sich ebenso hinsichtlich der Gedanken an den Tod findet, wie der von Tomer und Eliason (1996) beschriebene Zusammenhang von Todesangst und Schuldgefühlen. Hinzu kommt, dass Gedanken an den Tod mit einer höheren Aggressivität und Feindseligkeit einhergehen, was im Vollzugsalltag zu zusätzlichen Konflikten sowohl mit dem Personal als auch den Mitinhaftierten führen kann. Obwohl nicht eindeutig geklärt werden kann, ob die Gedanken an das Lebensende zur psychischen Beeinträchtigung führen oder umgekehrt, so kann doch angenommen werden, dass beide Aspekte eine Abwärtsspirale bilden und sich gegenseitig negativ beeinflussen. Für die Betreuung durch die Fachdienste lässt sich daraus ein höherer psychologischer Betreuungsbedarf in der belasteten Teilgruppe ableiten, der, wenn er subjektiv nicht ausreichend geboten wird, geringere Zufriedenheit nach sich zieht.

Allgemein liegen die Stärken der vorliegenden Studie in der relativ großen und umfangreichen Stichprobe, die zum Erhebungszeitpunkt nicht nur ein Siebtel aller österreichischen Inhaftierten im Alter ab 60 Jahren, sondern auch unterschiedliche Vollzugsformen umfasst und somit für eine gute Übertragbarkeit der Ergebnisse auf die Gesamtpopulation spricht. Nichtsdestotrotz gibt es auch einige Limitationen, die zu berücksichtigen sind. So ist aufgrund des Designs ein Selektionsbias anzunehmen, da für die Teilnahme eine umfassende Kenntnis der deutschen Sprache und eine gewisse kognitive Leistungsfähigkeit vorausgesetzt wurden, was eine mögliche Erklärung für die fehlenden Unterschiede hinsichtlich des MMST-Wertes bieten könnte. Auch erfolgte lediglich eine eindimensionale Erhebung des Vorliegens von Gedanken an den Tod. Zukünftige Untersuchungen, die Gedanken an das Lebensende und eine etwaige Todesangst differenziert erheben und mögliche allgemeine und altersspezifische Einflussfaktoren im Fokus haben, könnten daher wichtige Ergänzungen zu den bisherigen Erkenntnissen liefern.

Nichtsdestotrotz weisen die Ergebnisse der vorliegenden Untersuchung insgesamt darauf hin, dass Gedanken an den Tod und an das Sterben bei Inhaftierten ab 60 Jahren mit einer erhöhten psychischen Belastung einhergehen und dass vor allem Senioren, die bereits lang inhaftiert sind, mit der gedanklichen Auseinandersetzung mit einem möglichen Lebensende in Haft konfrontiert sind, woraus ein erhöhter psychologischer Betreuungsbedarf entsteht. Auch könnte eine Befragung zu Gedanken an den Tod in diesem Setting zukünftig ein ökonomisches Screening hinsichtlich psychischer Belastungen in dieser Altersgruppe darstellen.

Funding Open access funding provided by University of Vienna.

Interessenkonflikt N. Meuschke und R. Jagsch geben an, dass kein Interessenkonflikt besteht.

Open Access Dieser Artikel wird unter der Creative Commons Namensnennung 4.0 International Lizenz veröffentlicht, welche die Nut- 
zung, Vervielfältigung, Bearbeitung, Verbreitung und Wiedergabe in jeglichem Medium und Format erlaubt, sofern Sie den/die ursprünglichen Autor(en) und die Quelle ordnungsgemäß nennen, einen Link zur Creative Commons Lizenz beifügen und angeben, ob Änderungen vorgenommen wurden.

Die in diesem Artikel enthaltenen Bilder und sonstiges Drittmaterial unterliegen ebenfalls der genannten Creative Commons Lizenz, sofern sich aus der Abbildungslegende nichts anderes ergibt. Sofern das betreffende Material nicht unter der genannten Creative Commons Lizenz steht und die betreffende Handlung nicht nach gesetzlichen Vorschriften erlaubt ist, ist für die oben aufgeführten Weiterverwendungen des Materials die Einwilligung des jeweiligen Rechteinhabers einzuholen.

Weitere Details zur Lizenz entnehmen Sie bitte der Lizenzinformation auf http://creativecommons.org/licenses/by/4.0/deed.de.

\section{Literatur}

Aday RH (2006a) Aging prisoners. In: Berkman B (Hrsg) Handbook of social work in health and aging. Oxford University Press, Oxford, S 231-241

Aday RH (2006b) Aging prisoners' concerns toward dying in prison. Omega (Westport) 52:199-216. https://doi.org/10.2190/CHTDYL7T-R1RR-LHMN

Adbel-Khalek AM (2005) Death anxiety in clinical and non-clinical groups. Death Stud 29(3):251-259. https://doi.org/10.1080/ 07481180590916371

Adbel-Khalek AM, Neimeyer RA (2017) Death anxiety scale. In: Zeigler-Hill V, Shackelford T (Hrsg) Encyclopedia of personality and individual differences. Springer, Berlin, Heidelberg, New York, S 1-4 https://doi.org/10.1007/978-3-319-28099-8_21-1

Allgöwer R (2013) Senioren hinter Gittern. Strafvollzug in Baden-Württenmberg. Stuttgarter Zeitung. http://www.stuttgarterzeitung.de/inhalt.strafvollzug-in-badenwuerttemberg-seniorenhinter-gittern.9e57de35-d7fa-4777-9b81-5c40d73b8358.html. Zugegriffen: 13. Febr. 2016

American Civil Liberties Union (ACLU) (2012) At America's expense: the mass incarceration of the elderly. American Civil Liberties Union, New York

Ast A (2015) Die Seniorenstation der JVA Waldheim. FS 64:13-15

Baidawi S, Trotter C (2015) Psychological distress among older prisoners: a literature review. J Forensic Soc Work 5:234-257. https:// doi.org/10.1080/1936928X.2015.1075166

Baidawi S, Turner S, Trotter C, Browning C, Collier P, O'Connor D et al (2011) Older prisoners-A challenge for Australian corrections. Trends Issues Crime Crim Justice 426:1-11 (https://aic.gov. au/publications/tandi/tandi426)

Berger-Zell C (2019) Wenn ältere Menschen im Gefängnis leben. Bewährungshilfe 66:367-371

Bock C (2018) Senioren hinter Gittern [Dokumentation]

Borkenau P, Ostendorf F (1993) NEO-Fünf-Faktoren Inventar (NEOFFI) nach Costa und McCrea. Handlungsanweisung. Hogrefe, Göttingen

Brägger B (2018) Sterben hinter Gittern - Eine Analyse des rechtlichen Rahmens zur Frage des Ablebens im Freiheitsentzug. SZK 2:3-13

Cohen J (1988) Statistical power analysis for the behavioral sciences. Erlbaum, Hillsdale

Creavin ST, Wisniewski S, Noel-Storr AH, Trevelyan CM, Hampton T, Rayment D et al (2016) Mini-Mental State Examination (MMSE) for the detection of dementia in clinically unevaluated people aged 65 and over in community and primary care populations. Cochrane Database Syst Rev. https://doi.org/10.1002/14651858. CD011145.pub2
Cully JA, LaVoie D, Gfeller JD (2001) Reminiscence, personality, and psychological functioning in older adults. Gerontologist 41:89-95. https://doi.org/10.1093/geront/41.1.89

Devolder K (2016) Euthanasia for detainees in Belgium: the case of Frank Van den Bleeken. Camb Q Healthc Ethics 25:384-394. https://doi.org/10.1017/S0963180116000037

Franke GH (2000) BSI. Brief Symptom Inventory - Deutsche Version. Manual. Beltz, Göttingen

Frazier PH, Foss-Goodman D (1989) Death anxiety and personality: are they truly related? Omega (Westport) 19:265-274. https://doi. org/10.2190/141T-Q32F-LPPD-EY3W

Friedrichs H (2012) Sie nennen es „Röchelabteilung“. Wie Häftlinge altern - Eindrücke von einer Weihnachtsfeier in der Justizvollzugsanstalt Detmold. Die Zeit. https://www.zeit.de/2012/52/ Seniorentrakt-JVA-Gefaengnis. Zugegriffen: 13. Febr. 2016

Galt CP, Hayslip B (1998) Age differences in levels of overt and covert death anxiety. Omega (Westport) 37(3):187-202. https://doi.org/ 10.2190/U0M0-BWMV-WF6F-U4DH

Ghanem C, Kenkmann A (2019) Psychosoziale Unterstützungsangebote für lebensältere Menschen in Haft - Eine Literaturanalyse. Bewährungshilfe 66:320-344

Given JE, Range LM (1990) Life satisfaction and death anxiety in elderly nursing home and public housing residents. J Appl Gerontol 9(2):224-229. https://doi.org/10.1177/073346489000900208

Graber B, Hotz E, Holenstein P (2014) Senioren im Strafvollzug. Kriminalistik 12:750-758

Handtke V, Wangmo T (2014) Aging prisoners' views on death and dying: contemplating end-of-life in prison. J Bioeth Inq 11:373-386. https://doi.org/10.1007/s11673-014-9548-x

Hautzinger M, Keller F, Kühner C (2009) BDI-II. Beck-DepressionsInventar, 2. Aufl. Pearson Assessment, Frankfurt

Hayes AJ, Burns A, Turnbull P, Shaw JJ (2013) Social and custodial needs of older adults in prison. Age Ageing 42:589-593. https:// doi.org/10.1093/ageing/aft066

Hayslip B (2003) Death denial. In: Bryant CD, Peck DL (Hrsg) Handbook of death and dying. SAGE, London, S 34-42 https://doi.org/ 10.4135/9781412914291

Hirschbeck A (2013) Wenn Menschen im Gefängnis alt werden. Die Welt. http://www.welt.de/regionales/koeln/article112400998/ Wenn-Menschen-imGefaengnis-alt-werden.html. Zugegriffen: 13. Febr. 2016

Hostettler U, Marti I, Richter M (2019) Sterben im Justizvollzug - Juristische, ethische und praktische Möglichkeiten und Herausforderungen. Bewährungshilfe 66:356-366

Hostettler U, Richter M, Queloz N (2017) Lebensende im Gefängnis Rechtlicher Kontext, Institutionen und Akteure. SZK 16:18-20

Jastrzebski J, Slaski S (2011) Death anxiety, locus of control and big five personality traits in emerging adulthood in Poland. Psychol Educ J 48(4):1-15

Kenkmann A, Erhard S, Maisch J, Ghanem C (2020) Altern in Haft - Angebote für ältere Inhaftierte in der Bundesrepublik Deutschland. KrimOJ 1:101-122. https://doi.org/10.18716/ojs/krimoj/ 2020.1.7

Leigey ME (2015) The forgotten men: serving a life without parole sentence. Rutgers University Press, Chicago

Lob-Hüdepohl A (2019) Altern und Sterben im Gefängnis - Ethische Probleme in hochkomplexen Wirklichkeiten. Bewährungshilfe 66:293-307

Marti I (2015) Lebensende im Gefängnis: Vorstellungen, Ängste und Hoffnungen von Gefangenen im geschlossenen Vollzug in der Schweiz. In: Queloz N, Noll T, von Mandach L, Delgrande N (Hrsg) Verletzlichkeit und Risiko im Justizvollzug. Beiträge und Dokumentation der 9. Freiburger Strafvollzugstage. Stämpfli, Bern, S 143-150

Marti I, Hostettler U, Richter M (2014) Sterben im geschlossenen Vollzug: Inhaltliche und methodische Herausforderungen für die Forschung. SZK 13:26-43. https://doi.org/10.7892/boris.88053 
Meuschke N (2018) Der Lebensabend im Gefängnis. In: Maelicke B, Suhling S (Hrsg) Das Gefängnis auf dem Prüfstand. Springer, Wiesbaden, S 403-422

Meyer L (2019) Eine empirische Perspektive auf die gesundheitliche Situation älterer Inhaftierter. Bewährungshilfe 66:308-319

Pesch M (2015) Suizidalität in sächsischen Justizvollzugsanstalten Einfluss von Haftdauer, Persönlichkeitsauffälligkeiten und Persönlichkeitsstörungen auf die Suizidalität von Inhaftierten. Dissertation, Technische Universität Dresden

Richter M, Hostettler U, Marti I (2017) Chronik eines angekündigten Todes: Trajektorien und Logiken am Lebensende im Schweizer Justizvollzug. SZK 16:21-30

Roshani K (2012) Relationship between religious beliefs and life satisfaction with death anxiety. Ann Biol Res 3(9):4400-4405

Shaw DM, Elger BS (2016) Assisted suicide for prisoners? Stakeholder and prisoner perspectives. Death Stud 40:479-485. https://doi. org/10.1080/07481187.2016.1177621

Szaniawski T (1998) Typy postaw wobec śmierci a osobowość [Types of attitudes towards death vs. personality]. PAT, Krakau
Taghiabadi M, Kavosi A, Mirhafez SR, Keshvari M, Mehrabi T (2017) The association between death anxiety with spiritual experiences and life satisfaction in elderly people. Electron physician 9(3):3980-3985. https://doi.org/10.19082/3980

Thalmann B, Monsch AU, Bernasconi F, Berres M, Schneitter M, Ermini-Fünfschilling D et al (1997) Die CERAD Neuropsychologische Testbatterie - Ein gemeinsames minimales Instrumentarium zur Demenzabklärung. Manual. Geriatrische Universitätsklinik, Basel

Tomer A, Eliason G (1996) Toward a comprehensive model of death anxiety. Death Stud 20(4):343-365. https://doi.org/10.1080/ 07481189608252787

Walcher M (2018) Recht auf Selbstmord im Gefängnis. GRIN, München

Willems A (2015) Euthanasia of detainee: granting a prisoner's request. CJM 102:16-17. https://doi.org/10.1080/09627251.2015. 1026563 